\title{
Influence of exercise adherence level on modifiable coronary heart disease risk factors and functional-fitness levels in middle-aged men
}

\author{
E. S. Wallace PhD, J. A. White PhD*, A. Downie FFOMt, G. Dalzell MD $\ddagger$ and D. Doran \\ BA $\S$ \\ Department of Sport and Leisure Studies and NIBEC, University of Ulster, County Antrim, Northern Ireland, \\ UK, *Department of Public Health Medicine and Epidemiology, University Hospital, Queen's Medical Centre, \\ Nottingham, UK, +Occupational Health, Safety, and Environmental Services, Northern Telecom Europe \\ Limited, London, UK, †Regional Medical Cardiology Centre, Royal Victoria Hospital, Belfast, Northern Ireland, \\ UK and §Physical Education Centre, Queen's University, Belfast, Northern Ireland, UK
}

\begin{abstract}
The study investigated the potential health benefits of two levels of short-term exercise intervention, compared with non-intervention, on selected modifiable coronary heart disease (CHD) risk factors and functional fitness states in middle-aged men. All subjects underwent medical screening and signed informed consent before carrying out a standardized graded treadmill walk which required exercise up to $85 \%$ of age-predicted maximal heart rate. The results of the test were used together with musculoskeletal fitness assessments, for the prescription of a personalized exercise programme lasting 14 weeks. In all, 55 subjects were classified by adherence into high (HA, $n$ $=20$ ), low (LA, $n=19$ ), or non-adherence (NA, $n=16$ ) groups according to the degree of documented participation in the programme based on standard criteria (American College of Sports Medicine 1978, 1990). In addition, the respective groups of subjects were classified according to other modifiable and non-modifiable CHD risk factors and compared by self-reported levels of activity and sport involvement as well as perceived body weight classification. The results indicated that there were more comprehensive improvements in functional fitness including significant gains in aerobic endurance capacity, muscular endurance and flexibility in the HA group compared with the LA and NA groups. However, there was little or no change in the modifiable CHD risk factors in any of the respective groups, although anthropometric indices of weight, body mass index (BMI), skinfolds and waist:hips ratio tended to decrease in the $H A$ and LA groups but increased marginally in the NA group. Resting systolic blood pressures declined slightly in all groups, but serum total cholesterol remained unchanged irrespective of adherence level. The findings suggest that while functional fitness status gains may arise from optimal adherence level to short-term exercise conditioning, these are not matched by health gains in terms of amelioration of modifiable CHD risk factors. However, the level of adherence may be related to the recognized presence of other known CHD risk factors.
\end{abstract}

Keywords: Exercise adherence, CHD risk factors, fitness, middle-aged men

Address for correspondence: Dr E. S. Wallace, Department of Sport and Leisure Studies and NIBEC, University of Ulster, County Antrim BT37 0QB, Northern Ireland, UK

(C) 1993 Butterworth-Heinemann Ltd

0306-3674/93/020101-06
The benefits of regular physical activity in the promotion of health-related fitness and the amelioration of coronary heart disease (CHD) risk factors has received much attention recently with a consensus of agreement among investigators concerning its importance. A meta-analysis of studies concerning CHD and physical activity ${ }^{1}$ suggested that the literature supported the inference that physical activity is inversely and causally related to the incidence of CHD. Furthermore, the study concluded that the relative risk of inactivity as a factor in the development of CHD appears to be of similar magnitude to that of hypertension, hypercholesterolaemia and smoking. Therefore it was suggested that in preventive health programmes designed to ameliorate $\mathrm{CHD}$ risk, regular physical activity should receive the same attention as other modifiable risk factor management techniques, such as the control of blood pressure, reduction of serum cholesterol and the elimination of smoking.

The benefits of exercise amelioration of modifiable $\mathrm{CHD}$ risk factors such as reductions in blood pressure and cholesterol levels have been widely reported in the literature ${ }^{2}$. The reduction of resting blood pressure after a single bout of exercise in both normal and hypertensive patients ${ }^{3}$, together with the reduction in submaximal exercise systolic blood pressure after exercise training ${ }^{4,5}$ suggest that exerciseinduced effects of blood pressure regulation may contribute towards a reduction in CHD risk by lowering of blood pressure levels ${ }^{2}$. Thus, exercise may serve as a preventive modality in the amelioration of the age-dependent rise in blood pressure as well as exerting its effects through a reduced frequency of arterial hypertension development ${ }^{6,7}$.

The influence of physical activity in the modification of blood lipid profiles as a potential for CHD risk factor reduction has also been acknowledged ${ }^{8}$. Various types of exercise regimens including longterm, low-level activity ${ }^{9}$, as well as shorter-term aerobic programmes ${ }^{10}$ and resistive exercise/circuit weight training routines ${ }^{11}$ have been reported to influence blood lipid levels favourably. However, the influence of body weight loss and dietary changes is 
thought likely to contribute to the beneficial effects observed in lipid profile modification and subsequent improvement in CHD risk status ${ }^{2}$.

The population of Northern Ireland has not shared the recent successes of North America, Australia and several European countries in the reduction of the incidence of CHD over the last two decades ${ }^{12}$. One of the reasons postulated to account for this significant decrease in the levels of CHD in these countries is the concomitant increase in habitual levels of physical activity and associated positive health behaviour. At least part of this increased participation in regular physical activity may be attributed to the enhanced public awareness of the relationship between health and fitness and the provision for fitness programmes in the workplace ${ }^{13-16}$. However, when the present study was conducted there were no such operational workplace programmes in Northern Ireland. Furthermore, there appeared to be a distinctly different social attitude towards health and well-being, as well as current practice, in terms of workplace provision of corporate health and wellness programmes by Northern Ireland's employers ${ }^{17}$, which together may limit the potential effectiveness of such programmes compared with the other countries. Therefore, the present investigation was designed to determine the effects of adherence levels to a supervised short-term, workplace exercise programme on selected modifiable CHD risk factors and functional fitness levels in a group of middle-aged men volunteers.

\section{Methods}

\section{Subjects}

In all, 55 middle-aged men volunteers participated in the study. The subjects were self-selected from the middle and upper management levels of a multinational telecommunications company located on the outskirts of Belfast, Northern Ireland. The group comprised men of different socioeconomic status social classes I and II professional and intermediate (OPCS). All subjects were screened initially by obtaining a medical history, a series of pulmonary function tests and an examination by the company medical officer. Written informed consent was obtained from each subject before participation in the investigation.

\section{Procedures}

On arrival at the laboratory, the subjects completed a detailed exercise history and current physical activity questionnaire. A $32 \mu \mathrm{l}$ fasting blood sample was drawn from the subject's finger to determine total cholesterol level (Reflotron system, Boehringer Mannheim, Lewes, UK), and resting blood pressure was obtained by a trained nurse using standard auscultatory techniques. A range of anthropometric measurements was then taken, including height and weight, skinfold thicknesses and estimated body fat percentage ${ }^{18}$, and body girth dimensions for determination of waist to hips ratio. The subject then sat while a standard V-5 electrocardiogram recording was obtained and blood pressures were subsequently obtained before the start of the exercise test, which was conducted by an exercise physiologist with a cardiologist in attendance. The usual range of resuscitation equipment was available, including a defibrillator.

The exercise test consisted of a walk on a treadmill (P. K. Morgan, Chatham, UK) at a freely chosen speed at zero grade for an appropriate period of time to familiarize the subjects with the test requirements. This was followed by a modified Bruce protocol ${ }^{19}$ which required the subject to walk at a fixed speed of $5.65 \mathrm{~km} \mathrm{hr}^{-1}$ at zero grade followed by an initial gradient increase of $5 \%$ with subsequent increases of $2.5 \%$ with each incremental period of 3 min duration. The chosen endpoint of the test was $85 \%$ of age-related maximal heart rate which was predetermined using a standardized method ${ }^{20}$. Exceptions to this criterion were cases where subjects displayed irregular electrocardiogram (ECG) patterns, abnormal blood pressure responses, or chose voluntarily to terminate the test prematurely. ECG recordings (Cardiokinetics Life Trace 18, Cardiokinetics, Salford, UK) and blood pressure measurements (auscultatory techniques) were obtained during the last $30 \mathrm{~s}$ of each incremental exercise period. Total time for walking as well as the incremental stage of the test termination were recorded as measures of endurance. Three additional specific physical fitness tests were then completed which were directed at musculoskeletal function, including grip strength, hip flexibility and abdominal endurance as outlined in the Eurofit test battery ${ }^{21}$. All subjects subsequently received a comprehensive confidential personal report detailing the findings from the various tests, along with a consultation with the programme director, and the company's medical officer where necessary.

\section{Intervention}

After initial assessment each subject was issued with a voluntary, individualized exercise prescription based on their performance during the initial exercise test. Unless otherwise contraindicated, each programme was designed to meet the criteria of the guidelines for aerobic conditioning 22 and the subsequent revision of these criteria ${ }^{23}$. A wide range of activities was offered including walking, running, bicycling, cycle-ergometry, rowing, simulated crosscountry skiing and resistance training using a multigym fixed weight system. The subjects were supervised closely during the 14-week period of the conditioning programme and were instructed to record their exercise activities accurately in terms of exercise mode, intensity, duration and frequency of each session.

The data collected on activity participation permitted subjects to be classified into groups depending on their level of adherence to the exercise programme. Adherence levels were determined using an equation developed for determination of a total activity metabolic index $(\mathrm{AMI})^{24}$ where the overall AMI is:

Total AMI $=\Sigma(I \times M \times F \times T)$

where $I$ represents intensity coded in METS (resting metabolic equivalent), $M$ the number of months, $F$ 
the frequency per month, and $T$ the duration of each activity or exercise session. The total AMI score is believed to be closely associated with total kilocalories expended on leisure-time physical activity, a value of particular relevance in weight control ${ }^{24}$, and thus exercise for health.

Three discrete levels of physical activity were then obtained, in terms of light, moderate, and heavy AMI which were equated with non-adherence (NA), low adherence (LA), and high adherence (HA) levels in the present study. The HA group demonstrated an activity index which satisfied the American College of Sports Medicine (ACSM) criteriaa ${ }^{22}$ for aerobic conditioning. However, since the time of completion of the present study the ACSM revised and updated the criteria for aerobic conditioning ${ }^{23}$. The implications of these revisions now indicate that the LA classification conformed to the necessary levels prescribed to maintain cardiorespiratory fitness ${ }^{22}$, while the HA group conformed to the level necessary both to maintain and advance cardiorespiratory fitness ${ }^{23}$. After the 14-week conditioning period, subjects underwent the same battery of tests completed before the intervention programme. Thus data were available for both pre- and post-intervention, which permitted an evaluation of the effects of participation in each adherence level category.

\section{Data analysis}

After detailed analysis of the activity records the subjects were divided among the three exercise adherence level groups of NA, LA and HA for both pre- and post-intervention analyses. The data obtained from the pre-intervention tests were analysed using a one-way analysis of variance to determine if any significant differences existed between the three combinations of paired groupings before the intervention was applied. Similarly, this procedure was

Table 1. Physical characteristics of subjects within each exercise adherence level group

\begin{tabular}{lllll}
\hline $\begin{array}{l}\text { Exercise } \\
\text { adherence level }\end{array}$ & $\begin{array}{c}\text { Age } \\
\text { (years) }\end{array}$ & $\begin{array}{c}\text { Height } \\
(\mathrm{cm})\end{array}$ & $\begin{array}{c}\text { Weight } \\
(\mathrm{kg})\end{array}$ & $\begin{array}{c}B M I \\
\left(\mathrm{~kg} \mathrm{~m}^{-2}\right)\end{array}$ \\
\hline NA $(n=16)$ & $42.7(8.9)$ & $174.8(7.4)$ & $79.0(14.8)$ & $25.6(4.3)$ \\
LA $(n=19)$ & $37.6(9.7)$ & $175.6(5.9)$ & $76.5(8.8)$ & $24.8(2.2)$ \\
HA $(n=20)$ & $41.3(10.7)$ & $174.8(8.8)$ & $80.1(9.4)$ & $26.2(2.3)$ \\
Total $(n=55)$ & $40.4(7.0)$ & $175.1(6.9)$ & $78.5(11.43)$ & $25.5(3.4)$ \\
\hline
\end{tabular}

Values are mean(s.d.) applied to the post-intervention data to evaluate effects of adherence level upon the variables under investigation. Each adherence level group data were further analysed using a repeated measures analysis of variance to determine within group differences before and after the intervention period, with a post hoc Scheffe test applied to determine specific mean differences contributing to significant $F$ ratios identified by the analysis of variance procedures.

\section{Results}

There were no significant differences in mean age, height and weight at the initiation of the exercise programme in the respective exercise adherence level groups (Table 1). However, the LA group tended to be slightly younger and taller but lower in body weight and body mass index (BMI), compared with the NA and HA groups.

The coronary risk profile of each adherence level group indicated that there was a relatively even match among groups in terms of CHD risk factors, with the notable exceptions of history of heart disease in the family and elevated total serum cholesterol, both of which were seen to be more frequent in the HA group (Table 2). Nevertheless, there were no contraindications to exercise programming displayed by individual members of the respective adherence level groups.

The initial subjective estimates by each exercise adherence level group in terms of reported exercise and physical activity (EPA) profiles, present physical fitness levels, and perceived versus actual body weight classifications revealed several interesting anomalies (Table 3 ). While the respective groups were reasonably well matched in terms of 'sedentary' and 'moderately active' representations, few subjects considered themselves to be 'active' or 'highly active' - categories commensurate with minimal levels necessary for maintaining ${ }^{22}$ or enhancing ${ }^{23}$ aerobic fitness status. Furthermore, in the NA group physical fitness perceptions were at variance with the reported EPA profiles, whereby the majority of subjects considered themselves to be 'moderately active', while the distribution between 'very unfit' and 'moderately fit' was even. In the HA and LA groups there was a sequential rise in the 'moderately fit' to 'unfit' ratio, respectively, and the self-reported fitness level profiles closely matched the EPA perceptions. However, none of the subjects in any group regarded themselves as 'trained', although the

Table 2. Coronary risk profiles of the respective exercise adherence level groups

\begin{tabular}{|c|c|c|c|c|c|c|c|c|c|}
\hline $\begin{array}{l}\text { Exercise adherence } \\
\text { level }\end{array}$ & $\begin{array}{c}\text { CHD diag } \\
\text { (n) }\end{array}$ & $\begin{array}{l}\text { CHD family } \\
\text { (n) }\end{array}$ & $\begin{array}{l}\text { CHD death } \\
\text { (n) }\end{array}$ & $\begin{array}{l}\text { Rheum fever } \\
\text { (n) }\end{array}$ & $\begin{array}{c}A b n E C G \\
(n)\end{array}$ & $\begin{array}{l}\text { Hypertensive } \\
\text { (n) }\end{array}$ & $\begin{array}{l}\text { Hypercholes } \\
\text { (n) }\end{array}$ & $\begin{array}{c}\text { Smoke } \\
(n)\end{array}$ & $\begin{array}{c}>35 \text { years } \\
\text { (n) }\end{array}$ \\
\hline $\begin{array}{l}\text { NA }(n=16) \\
\text { LA }(n=19) \\
\text { HA }(n=20)\end{array}$ & $\begin{array}{l}1 \\
1 \\
1\end{array}$ & $\begin{array}{r}6 \\
4 \\
13\end{array}$ & $\begin{array}{l}3 \\
5 \\
6\end{array}$ & $\begin{array}{l}1 \\
1 \\
1\end{array}$ & $\begin{array}{l}0 \\
0 \\
1\end{array}$ & $\begin{array}{l}2 \\
0 \\
3\end{array}$ & $\begin{array}{r}9 \\
6 \\
13\end{array}$ & $\begin{array}{l}3 \\
2 \\
2\end{array}$ & $\begin{array}{r}10 \\
9 \\
13\end{array}$ \\
\hline Total $(n=55)$ & 3 & 23 & 14 & 3 & 1 & 5 & 28 & 7 & 32 \\
\hline
\end{tabular}

$n$, number; CHD diag, coronary heart disease previously diagnosed; CHD family, history of coronary heart disease in immediate family; CHD death, premature death in immediate family due to coronary heart disease; Rheum fever, previously had rheumatic fever; Abn ECG, abnormal electrocardiogram previously diagnosed; Hypercholes, hypercholesterolaemia; smoke, regular cigarette smoker 
Exercise adherence, CHD risk factors and fitness in men: E. S. Wallace et al.

Table 3. Reported levels of physical activity, physical fitness and body weight perceptions of the respective exercise adherence level groups

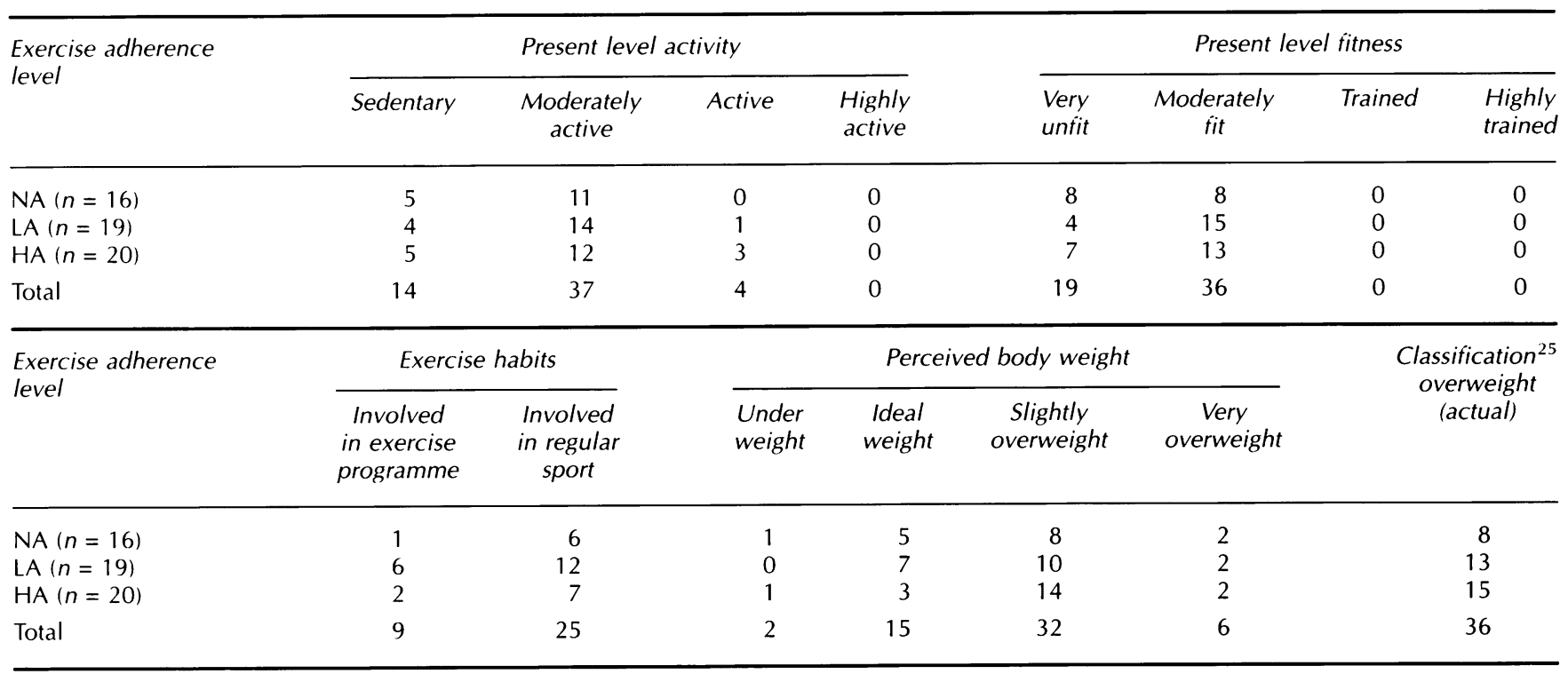

LA group indicated involvement in exercise and regular sports participation more frequently than either the NA or HA groups. Finally, in terms of perceived body weight classifications, few considered themselves 'underweight', while the distribution of 'overweight' was similar and the numbers considered to be 'ideal weight' formed a minority across the respective groups. However, a majority of subjects within each respective group considered themselves to be 'slightly overweight', which corresponded closely with those classified over $10 \%$ ideal body weight $^{25}$.
In general the results observed among the three levels of adherence to the 14-week aerobic conditioning programme indicated selective improvements in some functional fitness variables with little or no change in the modifiable CHD risk factors (Table 4). In the respective LA and $\mathrm{HA}$ groups there were significant increases of $13 \%$ and $43 \%$ in treadmill exercise times (both $P<0.001$ ) and concomitantly, $7 \%$ and $13 \%$ in the number of treadmill stages completed (both $P<0.05$ ), but no significant change in either measure in the NA group. Furthermore, at the pre-programme test the LA group had significantly

Table 4. The influence of exercise adherence level to aerobic conditioning on modifiable CHD risk factors and functional fitness levels

\begin{tabular}{|c|c|c|c|c|c|c|}
\hline \multirow[t]{3}{*}{ Variables } & \multicolumn{6}{|c|}{ Groups } \\
\hline & \multicolumn{2}{|c|}{$N A(n=16)$} & \multicolumn{2}{|c|}{$L A(n=19)$} & \multicolumn{2}{|c|}{$H A(n=20)$} \\
\hline & Pre & Post & Pre & Post & Pre & Post \\
\hline \multirow[b]{2}{*}{ TM time (min) } & \multirow[b]{2}{*}{$13.6(2.4)$} & \multirow[b]{2}{*}{$14.0(2.7)$} & \multicolumn{2}{|c|}{$\longrightarrow \neq-$} & \multirow{2}{*}{$10.9(3.4)$} & \multirow{2}{*}{$\overline{15.9(3.3)}$} \\
\hline & & & $14.6(4.3)$ & $16.5(4.3)$ & & \\
\hline TM stage (No.) & $4.6(0.7)$ & $4.5(1.6)$ & $4.6(1.0)$ & $5.1(1.1)$ & $3.7(1.1)$ & $5.0(1.0)$ \\
\hline $\mathrm{HR}$ (beats $\mathrm{min}^{-1}$ ) & $165.0(6.0)$ & $166.0(8.0)$ & $165.0(10.0)$ & $166.0(12.0)$ & $162.0(13.0)$ & $164.0(13.0)$ \\
\hline Syst BP (mmHg) & $129.0(14.7)$ & $123.0(16.3)$ & $120.0(10.9)$ & $118.0(11.6)$ & $125.0(18.6)$ & $123.0(12.2)$ \\
\hline Diast BP (mmHg) & $76.2(9.7)$ & $78.3(9.5)$ & $71.7(7.8)$ & $72.5(8.1)$ & $76.7(12.0)$ & $77.2(8.6)$ \\
\hline Weight (kg) & $79.0(14.8)$ & $79.4(14.4)$ & $76.5(8.8)$ & $75.8(8.5)$ & $80.2(9.4)$ & $79.0(9.5)$ \\
\hline $\mathrm{BMI}\left(\mathrm{kg} \mathrm{m}^{-2}\right)$ & $25.6(4.3)$ & $25.7(4.3)$ & $24.8(2.2)$ & $24.4(1.9)$ & $26.2(2.3)$ & $25.9(2.0)$ \\
\hline$\Sigma 4$ Skinfolds $(\mathrm{mm})$ & $49.6(16.4)$ & $52.1(16.7)$ & $44.6(15.0)$ & $43.0(10.7)$ & $45.7(12.4)$ & $43.9(9.2)$ \\
\hline Estimated body fat (\%) & $22.9(4.7)$ & $23.2(4.6)$ & $20.7(4.5)$ & $20.6(4.2)$ & $21.3(4.2)$ & $20.9(4.2)$ \\
\hline Waist: hips ratio & $90.1(5.8)$ & $91.5(5.7)$ & $91.1(5.0)$ & $90.6(4.4)$ & $92.1(4.7)$ & $90.7(4.3)$ \\
\hline Cholesterol $\left(\mathrm{mmoll}^{-1}\right)$ & $5.86(1.51)$ & $5.89(1.68)$ & $5.03(1.36)$ & $5.15(1.04)$ & $5.58(1.31)$ & $5.51(1.33)$ \\
\hline Grip strength (kg) & $52.9(6.8)$ & $52.8(6.3)$ & $52.7(8.5)$ & $52.6(7.10)$ & $54.3(6.70)$ & $52.7(6.10)$ \\
\hline $\begin{array}{l}\text { Abdominal endurance } \\
\text { (No. per } 30 \text { s) }\end{array}$ & $16.7(4.9)$ & $21.2(5.3)$ & $18.7(4.0)$ & $22.9(4.70)$ & $17.3(3.5)$ & $22.7(6.0)$ \\
\hline Flexibility (cm) & $7.7(4.8)$ & $7.2(5.4)$ & $5.7(6.8)$ & $5.6(6.9)$ & $6.9(4.8)$ & $8.6(6.1)$ \\
\hline
\end{tabular}

Syst BP, systolic blood pressure; Diast BP, diastolic blood pressure Significance levels: ${ }^{*} P<0.05 ;+P<0.01 ; \neq P<0.001$

Values are mean(s.d.) 
longer treadmill exercise times and number of treadmill stages completed (both $P<0.005$ ) than the HA group. However, while there were marginal post-programme decreases in end exercise heart rates and resting systolic and diastolic blood pressures in all three adherence level groups, these were not significant.

Similarly there were no significant anthropometric changes among the three levels of exercise adherence after the conditioning programme, although there was a trend towards a slight decrease in body weight, body mass index, sum of four skinfolds, estimated body fat percentage and waist:hips ratio in the LA and HA groups, which contrasted with the marginal increase in these measures in the NA group. However, serum total cholesterol levels remained unchanged over the duration of the study. Finally, significant improvements in abdominal endurance were observed in the NA group $(22 \%, P<0.05)$ and in the respective LA and HA groups (22\% and $31 \%$, both $P<0.01$ ) as well as an increase in flexibility in the HA group $(24 \%, P<0.05)$, but no significant change in grip strength in any group after the intervention programme.

\section{Discussion}

The present study investigated the responses of selected modifiable CHD risk factors, and functional fitness levels to a short-term (14-week) aerobic conditioning programme in relation to three levels of adherence by middle-aged men. The results suggested that in comparison with non-adherence (NA) and low adherence (LA), high adherence (HA) to a standard set of physical activity guidelines ${ }^{23}$ led to a more comprehensive improvement in a range of functional abilities including aerobic endurance, muscular endurance and flexibility. However, in terms of modifiable CHD risk factor status, while there were trends towards systolic blood pressure and body weight reductions associated with LA and $\mathrm{HA}$, there was no evidence to suggest changes in serum total cholesterol levels regardless of the level of adherence.

Enhanced aerobic endurance capacity was evidenced by significant increases in treadmill exercise times and the number of exercise stages completed by the LA and HA groups with the latter recording the largest improvement in both parameters after the conditioning programme. These findings are in agreement with increased endurance capacity observed in a similar population group who participated in an exercise programme using similar guidelines ${ }^{14}$. Furthermore, the increases in treadmill exercise performances by the LA and HA groups were accompanied by marginal but non-significant increases in end exercise heart rates which suggested an improvement in exercise tolerance of the respective groups of subjects.

The changes in anthropometric dimensions in general reflected the levels of adherence to the conditioning programme. Body weight changes showed a significant reduction in the HA group, a non-significant trend towards a reduction in the LA group, but little or no change in the NA groups over the 14-week period. These relatively modest body weight decreases of $2.4 \%$ and $2.0 \%$ in the HA and LA groups, respectively, were similar to those documented in previous studies ${ }^{14,26}$. However, even though body weight decreased most profoundly in the HA group, which exercised above the minimal standard guidelines ${ }^{23}$, the weight reduction observed may have been assisted by other factors such as dietary modification which was not assessed. Furthermore, the small but non-significant reduction in BMI noted in all groups was not accompanied by any discernible changes in the estimated percentage of body fat, which suggested that no change occurred in body composition in the respective groups.

In each of the three adherence level groups resting systolic blood pressure showed a tendency toward a small but non-significant reduction after the conditioning programme, while resting diastolic blood pressure remained relatively unchanged. However, it was considered unlikely that normotensive subjects such as those in the present study would benefit from exercise conditioning related blood pressure reductions observed in subjects with mild or borderline hypertension ${ }^{27,28}$. Serum total cholesterol levels remained relatively unaffected by the conditioning programme regardless of the level of exercise adherence. This suggested that, unlike other studies of longer duration, which have demonstrated significant reductions in cholesterol ${ }^{24,29}$, the duration of 14 weeks used in the present study was insufficient to modify total cholesterol levels. This conclusion is supported by the findings of other studies using exercise programmes of similar duration which failed to demonstrate significant changes in cholesterol level $^{26,30,31}$. It has been suggested, therefore, that exercise alone has little effect on total cholesterol level $^{31}$, although significant changes in other CHD risk factor lipid fractions e.g. high density lipoprotein (HDL): low density lipoprotein (LDL) cholesterol ratio may occur after exercise training without alteration in total cholesterol level ${ }^{11}$.

An evaluation of the initial coronary risk profile of the respective exercise adherence level groups (Table 2) indicated that the HA group had a relatively higher incidence of family CHD along with elevated serum total cholesterol level than the NA and LA groups. The presence of these factors therefore may have influenced the exercise participation level by the HA group given the recent publicity concerning the prevalance of CHD risk factors in Northern Ireland and its status as world leader in premature mortality from $\mathrm{CHD}^{12}$. Indeed, it has been suggested that willingness to take part in, along with type and amount of exercise performed, as well as adherence level to such programmes may be related to the presence of one or more CHD risk factors ${ }^{29}$. However, the level of adherence to the conditioning programme in the present study did not appear to be related to the subjective perceptions of level of activity, fitness status or personal body weight characteristics (Table 3), but may have been influenced by the involvement in similar programmes, other sport activities and the relatively overweight 
levels demonstrated by the LA and HA groups (Table 3).

In conclusion, the findings of the present investigation suggested that differences in exercise adherence level to a short-term (14-week) conditioning programme may selectively influence specific function levels of fitness and modifiable CHD risk factors. Furthermore, the results indicated that adherence to the minimum level of exercise suggested by standard guidelines ${ }^{23}$ produced fewer positive improvements in low adherence compared with high adherence, while the isolated change noted in non-adherence, for example improved muscular endurance, may have been attributable to habituation effects alone. Nevertheless, it was evident that the relatively short duration of the conditioning programme utilized by the present study produced relatively fewer beneficial effects when compared with similar programmes of longer duration. However, the reduced benefits of short-duration conditioning programmes must be set against the problems of adherence, maintenance, and drop-out rate associated with more prolonged timescales.

\section{Acknowledgements}

A special thanks is extended to all the subjects from Northern Telecom Europe, Monkstoun, Northern Ireland who volunteered to participate in this study.

\section{References}

1 Powell KE, Thompson PD, Casperson CJ, Kendrick JS. Physical activity and the incidence of coronary heart disease. Annu Rev Public Health 1987; 8: 253-87.

2 Fentem PH, Bassey EJ, Turnbull NB. The New Case for Exercise. London: The Sports Council and Health Authority, 1988.

3 Wilcox RG, Bennett T, Brown AM, MacDonald IA. Is exercise good for high blood pressure? $\mathrm{Br}$ Med J 1982; 285: 767-9.

4 Cade $\mathrm{R}$, Mars D, Wagemaker $\mathrm{H}$ et al. Effect of aerobic exercise training on patients with systemic arterial hypertension. $A m J$ Med 1984; 77: 785-90.

5 Duncan JJ, Farr JE, Upton SJ, Hagan RD, Ogles ME, Blair SN. The effects of aerobic exercise on plasma catecholamines and blood pressure in patients with mild essential hypertension. JAMA 1985; 254: 2609-13.

6 Blair SN, Goodyear NN, Gibbons LW, Cooper KH. Physical fitness and incidence of hypertension in healthy normotensive men and women. JAMA 1984; 252: 487-90.

7 Findlay IN, Taylor RS, Dargie HJ et al. Cardiovascular effects of training for a marathon run in unfit middle aged men. $\mathrm{Br}$ Med J 1987; 295: 521-4.

8 Royal College of Physicians. Medical Aspects of Exercise: Benefits and Risks. Report of the Royal College of Physicians, 1991.

9 Altina DA, Falorni P, Canepele N, Pieri V, Rossetti M. Biological and haematological modifications after two years of physical activity in middle aged men. J Sports Med 1981; 21: 253-8.
10 Haskell WL, Taylor HL, Wood PD, Schrott H, Heiss G. Strenuous physical activity, treadmill exercise test performance and plasma high-density lipoprotein cholesterol. The Lipid Research Clinics Program Prevalence Study. 1980; 62 (Suppl 4): 53-61.

11 Hurley BF, Hagberg JM, Goldberg AP et al. Resistive training can reduce coronary risk factors without altering $\dot{V}_{\mathrm{O}_{2} \max }$ or percent body fat. Med Sci Sports Exerc 1991; 20: 150-4.

12 Evans AE, McCrum EE, Patterson CC. 'Monica Project'. Belfast, Northern Ireland: Royal Victoria Hospital, 1990.

13 Durbeck DC, Heinzmann F, Haskell WL et al. The National Aeronautical and Space Administration - US Public Health Service Health Evaluation and Enhancement Programme. Am J Cardiol 1972; 30: 784-90.

14 Rhodes EC, Dunwoody DM. Physiological and attitudinal changes in those involved in an employee fitness programme. Can J Public Health 1980; 71: 331-6.

15 Blair SN, Piserchia PV, Wilbur CS, Crowder JH. A public health intervention model for worksite health promotion. JAMA 1986; 225: 921-6.

16 Klarreich SH. Health promotion in the workplace: a historical perspective. In: Klarreich, ed. Health and Fitness in the Work Place. London: Praeger, 1987.

17 Wallace ES, White JA, Scully D. Corporate health and wellness in Northern Ireland. Irish Business and Administration Research 1990; 11: 91-9.

18 Durnin JVGA, Wormersley J. Body fat assessed from total body density and its estimation from skinfold thickness. $\mathrm{Br} J$ Nutr 1974; 30: 27-9.

19 Bland PR, Williams PT. Activity patterns of men attending for fitness assessment. Br J Sports Med 1988; 22: 101-6.

20 Karvonen M, Kentala E, Mustala O. The effects of training on heart rate. A longitudinal study. Ann Med Exp Biol Fenn 1957; 35: 307-13.

21 Council of Europe. Handbook for the EUROFIT Test of Physical Fitness. 1988

22 American College of Sports Medicine. Position statement on the recommended quantity and quality of exercise for developing and maintaining fitness in healthy adults. Med Sci Sports Exerc 1978; 10: VII-X.

23 American College of Sports Medicine. Position statement on the recommended quantity and quality of physical activity to promote and maintain cardiorespiratory and muscular fitness in healthy adults. Med Sci Sports Exerc 1990; 22: 265-74.

24 Taylor HL, Jacobs DR Jr, Schucker B, Knudsen J, Leon AS, Debacker G. A questionnaire for the assessment of leisure time physical activities. J Chron Dis 1978; 31: 741-55.

25 Royal College of Physicians. Obesity: a report of the Royal College of Physicians. J R Coll Physicians Lond 1983; 17: 5-65.

26 Pauley J, Palmer J, Wright C, Pfeiffer G. The effects of a 14 week employee fitness programme on selected physiological and psychological parameters. J Occup Med 1982; 24: 457-63.

27 Kiyonaga A, Arakawa K, Tomaka H, Shindo M. Blood pressure and hormonal responses to aerobic exercise. Hypertension 1984; 7: 125-31.

28 Kukkomen K, Rauramaa R, Voutilainen E, Lansimies E. Physical training of middle-aged men with borderline hypertension. Ann Clin Res 1982; 14 (Suppl 34): 139-45.

29 Teraslinna P, Partanen T, Koskela A, Oja P. Characteristics affecting the willingness of executives to participate in an activity programme aimed at coronary heart disease prevention. J Sports Med 1969; 9: 224-9.

30 Lindner $\mathrm{CW}$, Durant $\mathrm{RH}$, Mahoney OM. The effect of physical conditioning on serum lipids and lipoproteins in white male adolescents. Med Sci Sports Exerc 1983; 15: 232-6.

31 Gilliam TB, Freedson PS. Effects of a 12 week school physical fitness program on peak $\dot{V}_{\mathrm{O}_{2}}$ body composition and blood lipids in 7 to 9 year old children. Int J Sports Med 1980; 1: 73-8. 